\title{
The Design and Simulation of HPLC's Structure
}

\author{
Zening $\mathrm{Xu}^{1, \mathrm{a}}$, Xiaoxia Wang ${ }^{1, \mathrm{~b},}$,Liang Hou ${ }^{1}$ and Leigang $\mathrm{Liu}^{2}$ \\ ${ }^{1}$ Liaoning University of Science and Technology, Anshan, Liaoning114051, China \\ ${ }^{2}$ The wire factory of Anshan Iron and Steel company stock, Liaoning, China \\ axuzening-1@163.com, bwangxiaoxiahhh@126.com
}

Keywords: Chromatographic Column; ANSYS CFX fluid simulation; Structure Optimization

\begin{abstract}
As an important part of chromatography instruments, chromatographic column plays an important role in the progress of science and the development of production. In order to avoid the traditional process , design - processing- test - Fixed design - re-processing and effectively shorting the design cycle save a lot of manpower, material and financial resources. This paper offers to watch the changes of flow profile within the column by the application of simulation software ANSYS CFX .Besides, according to the distribution of flow profile, the traditional column has been done a lot of dynamic simulation, especially the stigma part of the column. From a fluid Machinery perspective, we simulated and designed the structure, and then found out the best separation efficiency of the column structure.
\end{abstract}

\section{Introduction}

Matography is a modern separation method. In addition, it's also a good tool to study physics and chemistry ${ }^{[1]}$. The figure shows the basic structure of chromatographic column:

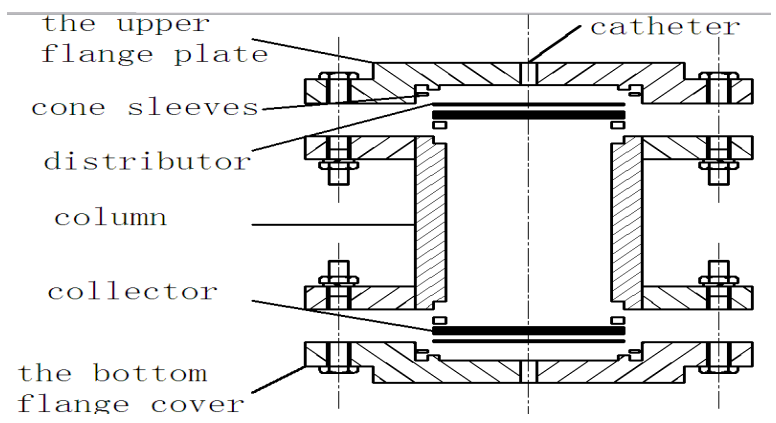

Fig.1. Chart of traditional structure of column

\section{The flow profile of fluid within the chromatographic column}

The structure of Chromatographic column is simplified to the flow of fluid in the pipe. Close to the wall, there is the thinnest laminar flow layer. And the fluid makes laminar flow movement within the laminar flow layer. From the bottom to the pipe center, the farther away from the wall, the smaller will the wall affect the fluid. Passing a transition layer, the fluid capacity of the hybrid papers enhanced and formed a fully turbulent zone, as shown in Fig.1. The calculating Formula about the thickness $\delta$ of the laminar flow layer:

$$
\delta=\frac{34.2 d}{\operatorname{Re}^{0.875}}
$$


Eq.1 shows that the thickness $\delta$ of the laminar flow layer decreases with Reynolds number's increase me.

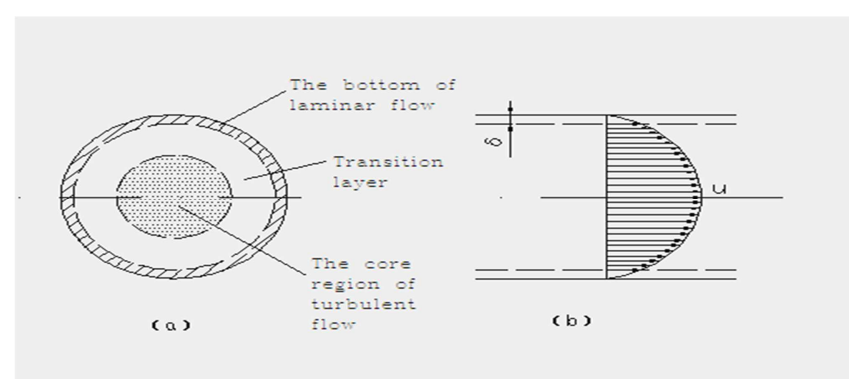

Fig.2. Turbulent structure and velocity distribution:

(a) Turbulence structure; (b) velocity distribution

\section{Computational fluid dynamics theory of Chromatographic column}

The fluid motion equation--NS ( Navier -Stokes) equation and the continuity equation as a non-steady state serve as the basic equation of turbulent motion.

Presently, there is no swirling flow and the buoyancy calculation for the flow of the column within the flow channels, so selecting the two equation $k-\varepsilon$ model is more reasonable. Analysis in the near wall region, we use wall-function method.

Reynolds stress in the standard $k-\varepsilon$ model is:

$$
-\rho \overline{u_{i}^{\prime} u_{j}^{\prime}}=\mu_{T}\left(\frac{\partial u_{i}}{\partial u_{j}}+\frac{\partial u_{j}}{\partial u_{i}}\right)-\frac{2}{3} \rho k \delta_{i j}
$$

Eddy viscosity is:

$$
\mu_{T}=C_{\mu} \rho \frac{k^{2}}{\varepsilon}
$$

Reynolds stress in which introduces new variables $k$ (turbulent kinetic energy), $\varepsilon$ (dissipation rate), with a model $k-\varepsilon$ to be closed. Precise $k-\varepsilon$ equation is introduced through the N-S equations, been simplified and got Eq.4 and Eq.5.

Equation $k$ :

$$
\frac{\partial(\rho k)}{\partial t}+\frac{\partial\left(\rho u_{j} k\right)}{\partial x_{j}}=\frac{\partial}{\partial x_{j}}\left[\left(\mu+\frac{\mu_{t}}{\sigma_{k}}\right) \frac{\partial k}{\partial x_{j}}\right]+\rho p_{k}-\rho \varepsilon
$$

Equation $\varepsilon$ :

$$
\begin{aligned}
& \frac{\partial(\rho \varepsilon)}{\partial t}+\frac{\partial\left(\rho u_{j} \varepsilon\right)}{\partial x_{j}}=\frac{\partial}{\partial x_{j}}\left[\left(\mu+\frac{\mu_{t}}{\sigma_{\varepsilon}}\right) \frac{\partial \varepsilon}{\partial x_{j}}\right]+\rho C_{1} \frac{\varepsilon}{k} \mathrm{p}_{\mathrm{k}}-\rho \mathrm{C}_{2} \frac{\varepsilon^{2}}{k} \\
& k=\frac{\overline{u_{i}^{\prime} u_{i}^{\prime}}}{2} \text { is the turbulent kinetic energy, } \quad \varepsilon=V \overline{\frac{\partial u_{i}^{\prime}}{\partial x_{j}} \frac{\partial u_{i}^{\prime}}{\partial x_{j}}} \text { is the turbulent energy dissipation, } \mu_{t}=\rho C_{\mu} \frac{k^{2}}{\varepsilon}
\end{aligned}
$$

is the eddy viscosity, $p_{k}=\frac{\mu_{t}}{\rho}\left(\frac{\partial u_{i}}{\partial x_{j}}+\frac{\partial u_{j}}{\partial x_{i}}\right) \frac{\partial u_{i}}{\partial x_{j}}$ is the production term for the turbulent kinetic energy, in which the subscript $j$ can use 1,2,3 and means spatial coordinates $\mathrm{x}, \mathrm{y}, \mathrm{z}$, the $j$ footnote which is the same sails that the three sums.

If the Reynolds stress calculates according to Eq.3 Boussinesq eddy viscosity model to, $k, \varepsilon$ equation, Eq.2 and the continuity equation forms a complete closed turbulent flow equations.

\section{The Design of HPLC' three-dimensional structure}

Applying the three-dimensional modeling software of PRO / E, we established the mathematical model of fluid within HPLC column. We watched the speed distribution and the time distribution of the flow profile, optimized the structure of the HPLC column with ANSYS CFX software. 


\section{Fluid's mathematical model and flow profile's simulation within chromatographic column}

The boundary conditions of fluid within chromatographic column. 1. Inlet boundary conditions: there is given stability pressure and flow on Inlet boundary. According to working conditions, the pressure along the direction of the column center line is set to 100atm; the velocity which is perpendicular to the column centerline is zero. 2 . Wall conditions: the wall surface adopts the equilibrium condition, the wall function. 3. Outlet boundary conditions: the turbulent flow achieves a relative balance in the export of the column, the gradient of velocity and turbulent kinetic energy in the mainstream direction is zero. Outlet pressure is set to an atmospheric pressure.

The Simulation and optimization of fluid within chromatographic column. As the boundary conditions of various parts are different, This paper uses software ICEM CFD to mesh and optimist the grid on the column, distributor, and some catheters ${ }^{[2]}$, the grid sets relatively dense in the important part or the greater gradient part, and sparse in the unimportant part .

Firstly, this paper made a simulation of the two point injection mode, and then simulated the side injection of several structures, including the sample injected by central single point injection mode, the sample injected by two points within the isotropy tangential line, the sample injected by two point within symmetric tangential line ${ }^{[3]}$.

This paper adopted the way of center entrance and changed distributor surface into a Conical plane with certain cone angle ${ }^{[4]}$, as shown in Fig.3, the sample solution from the catheter uniformly dispersed in the cone and maintained the same flow rate, so reduced the positive impact force which the solution acts on the distributor, the flow of the solution is more rules, for which we did some simulations for different angles $\theta$.

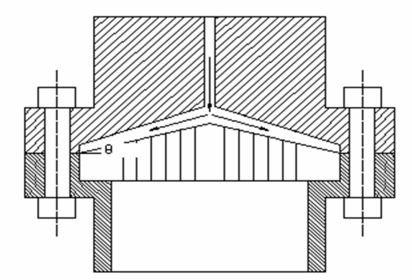

Fig.3. Hart of the improved distributor

When $\theta=1^{\circ}$, the flow profiles within chromatographic column are flowing:

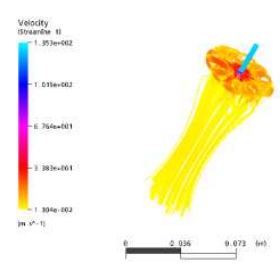

Fig.4. Velocity streamline through the column $\theta=1^{\circ}$

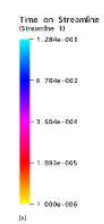

Fig.5. Time on streamline through the column $\theta=1^{\circ}$

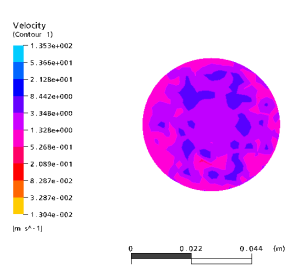

Fig.6. $1^{\circ}$ Velocity on the distributor $\theta=1^{\circ}$

From the simulation results, after changed distributor surface into a Conical plane with certain cone angle, the fluid profile of column has been greatly improved, but exit velocity of distributor was still quite irregular. So we made simulation about a large cone angle $\theta=10^{\circ}$, We compared the simulations about $\theta=1^{\circ}$ and $\theta=10^{\circ}$, when $\theta=10^{\circ}$, the flow lines of column were more evenly distributed, and time followed linear distribution.

The flow profile took $\theta=2^{\circ}, 3^{\circ}, 4^{\circ}$ respectively, from the observed, when $\theta=2^{\circ}$, the fluid flow was relatively stable, and the exit velocity of distributor also was more uniform, it can be said that $\theta$ $=2^{\circ}$, the flow structure was the best. 


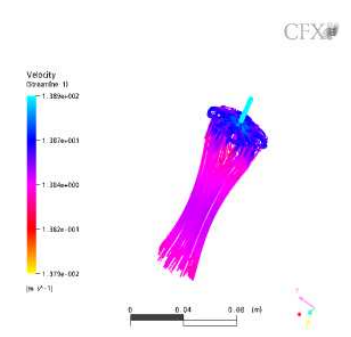

Fig. $7.10^{\circ}$ Velocity streamline through the column
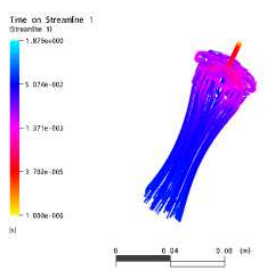

Fig. $8.10^{\circ}$ Time one streamline through the column

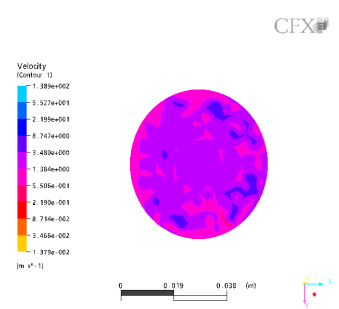

Fig.9. Velocity on the distributor $\theta=10^{\circ}$

The original diversion holes on the distributor has been re-refined and distributed, the sample solution more fully and evenly was injected into the column stationary phase, the simulation results were shown in Fig.10 to Fig.12.

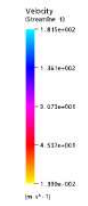

Fig. $10 . \theta=2^{\circ}$ Velocity streamline through the column after Refinement

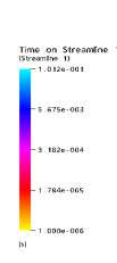

Fig.11. $\theta=2^{\circ}$ Time one streamline through the column after Refinement

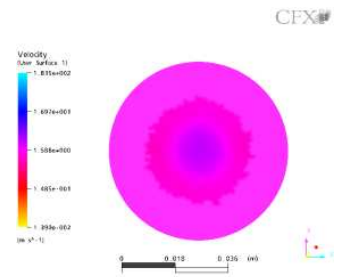

Fig.12. $\theta=2^{\circ}$ the exist Velocity of distributor which is $90 \mathrm{~mm}$ away from distributor

From the simulation results, the sample solution fully and evenly was injected into the column after re-refined and distributed the original diversion holes on the distributor ${ }^{[5]}$, the exit of column was defined at $90 \mathrm{~mm}$ away from distributor, the flow distribution of the sample solution in such a structure was uniform and stable.

\section{Summary}

(1) According to the simulation above, if the distributor's design is improper, two conditions often occur. The sample is concentrated in the center of column, It can not spread to the wall, there is the kind of situation that the flow model is the center point. The other is that the sample has uneven distributed in the cross section of stigma, the sample locally is too thick, there are irregular flow profiles.

(2)The simulation concludes the relationship between Distributor's cone angle and the flow Pattern, and ultimately determine that the distributor of $\theta=2^{\circ}$ is the best structure; Analysis the velocity distribution at each section on the analytical column, when the column length is $90 \mathrm{~mm}$, the fluid flow is the best and the separation efficient is the highest.

\section{References}

[1] Ruonong Fu . Introduction to chromatographic analysis. Beijing: Chemical Industry Press, 2005.

[2] Fujun Wang. Computational Fluid Dynamics-CFD software principles and applications. Beijing:Qinghua University Press, 2004.

[3] Hong Xu, Hou Jian. The type of HPLC column and the selection method of mobile phase. Measurement and Testing Technology, 2010.

[4] Dong QiangLu.Shi QiangDai.Surface and inerfacial gravity waves due to a disturbance steadily moving in a two-layer inviscid fluid. ScienceDirect Journal of Hydrodynamics,2010,22(5):40-44.

[5] Erik.H. \& Edit.F. \& K.Agnes,Effect of long-term storage and use on the properties ofreversed-phase liquid surfactant.Talanta,2008(76)172-182. 